J. Clin. Chem. Clin. Biochem.

Vol. 26, 1988, pp. 229-231

(C) 1988 Walter de Gruyter \& Co.

Berlin - New York

\title{
Bestimmung von Tri- $n$-butylphosphat in Plasmapräparaten nach Festphasenextraktion und Kapillar-Gaschromatographie
}

\author{
Von P. Pfeiffer \\ Institut für Mikrobiologie und Weinforschung, Johannes Gutenberg-Universität, Mainz
}

(Eingegangen am 24. November 1987/26. Januar 1988)

Zusammenfassung: Es wird eine einfache Methode zur quantitativen Bestimmung von Tri-n-butylphosphat in Blutplasma-Präparaten beschrieben. Die Probe wird über eine Octadecyl-Extraktionssäule gegeben, von der Tri- $n$-butylphosphat mit Chloroform eluiert wird. Durch Kapillar-GC können $50 \mu \mathrm{g} / 1$ Tri- $n$-butylphosphat bei einer Wiederfindungsrate von über $90 \%$ nachgewiesen werden.

Determination of tri-n-butylphosphate in plasma preparations using solid phase extraction and capillary gus chromatography

Summary: A simple method for the quantitative determination of tri- $n$-butylphosphate in blood plasma preparations is described. The sample is passed through an octadecyl extraction column from which tri-nbutylphosphate is eluted with chloroform. By capillary GC $50 \mu \mathrm{g} / \mathrm{l}$ tri-n-butylphosphate can be detectcd with a recovery of more than $90 \%$.

\section{Einführung}

Das Risiko der Übertragung von Viren durch Blut und Plasmaprodukte macht es notwendig, nach effektiven Verfahren zu suchen, um vor allem Hepatitisviren (Hepatitis B-Virus, Hepatitis Non-A, Non-BVirus) und das Human Immunodeficiency Virus (HIV) zu inaktivieren. Für primär nicht sterilisierbare Produkte wurden verschiedene, leider oft nur zum Teil befriedigende Techniken entwickelt, wie z. B. Erhitzen $(1,2)$, Behandlung mit $\beta$-Propiolacton und UV-Licht $(3,4)$ und Anwendung verschiedener Detergentien oder organischer Lösungsmittel (5). Als besonders schonend und zugleich wirkungsvoll erwies sich zur Virusinaktivierung die Behandlung mit Tri- $n$-butylphosphat in Kombination mit dem Detergenz Natriumcholat. Das wasserunlösliche Tri-n-butylphosphat bildet mit dem Cholat eine Emulsion, die dem Blutpräparat in geringen Mengen zugegeben wird und lipophile Viren inaktiviert. Beide Verbindungen wer- den nach ihrer Einwirkung durch Dialyse an Membranen oder Sephadex weitgehend abgetrennt $(6,7)$. Wegen der Toxizität von Tri-n-butylphosphat ist es notwendig, die Restkonzentration dieses Stoffes in behandelten Blutpräparaten zu bestimmen.

Es soll hier ein einfaches und effektives Extraktionsverfahren für Tri- $n$-butylphosphat aus wäßrigen Lösungen und die anschließende gaschromatographische Bestimmung an einer Kapillarsäule beschrieben werden.

\section{Material und Methoden}

Herkunft der Proben

Plasmapräparate, die zum Teil mit Tri-n-butylphosphat und Natriumcholat behandelt waren, wurden vom DRK-Blutspendedienst Rheinland-Pfalz, Bad Kreuznach, zur Verfügung gestellt. 


\section{Herkunft der Chemikalien}

Tri-n-butylphosphat p. A. von Merck, Darmstadt; Natriumcholat von Serva, Heidelberg; Tri- $n$-pentylphosphat von Alfa-Ventron, Karlsruhe; Chloroform p. A. und Methanol p. A. von Roth, Karlsruhe.

\section{Geräte und Säulen}

Festphasenextraktionssystem 10 SPE von Baker, Groß-Gerau; Bond Elut $\mathrm{C}_{18}$-Extraktionssäulen mit Polyethylenfritten und $500 \mathrm{mg}$ Packungsinhalt von ICT, Frankfurt; Gas-Chromatograph Modell 3700 mit FID von Varian, Darmstadt; CR-1BIntegrator von Shimadzu, Duisburg; Durabond-Wax-Kapillare, $30 \mathrm{~m}, 0,32 \mathrm{~mm}$ i. D., 0,25 $\mu \mathrm{m}$ Filmdicke von ICT, Frankfurt; WG11 Weichglas-Kapillare $25 \mathrm{~m}, 0,25 \mathrm{~mm}$ i. D., 0,2 $\mu \mathrm{m}$ Filmdicke von WGA, Pfungstadt.

\section{Extraktionsbedingungen}

Durch eine $\mathrm{C}_{18}$-Säule mit $500 \mathrm{mg}$ Inhalt wurden $2 \times 3 \mathrm{ml}$ Methanol und anschließend $2 \times 3 \mathrm{ml}$ destilliertes Wasser gesaugt, ohne daß die Säule trocken lief. Danach wurden $4 \mathrm{ml}$ behandeltes Plasma und $10 \mu \mathrm{l}$ interner Standard $(100 \mathrm{mg}$ Trin-pentylphosphat, gelöst in $10 \mathrm{ml}$ Choroform) auf die Säule gegeben und mit $2 \times 3 \mathrm{ml}$ destilliertem Wasser nachgewaschen. Zur Entfernung des Wassers wurde die Säule 20 Minuten unter Vakuum getrocknet. Die absorbierten Substanzen wurden mit $2 \times 300 \mu \mathrm{l}$ Chloroform eluiert, welches bis zur Trockene bei Raumtemperatur abgedampft wurde. Der Rückstand wurde in $20 \mu \mathrm{l}$ Chloroform aufgenommen und gaschromatographisch analysiert.

\section{Chromatographische Testbedingungen}

Säulenvordruck 0,7 bar $N_{2}$, Split 1:60, Einspritzvolumen 0,1 $\mu l$, Injektor $250^{\circ} \mathrm{C}$, Säulenofen $190^{\circ} \mathrm{C}$, Detektor $250^{\circ} \mathrm{C}$.

\section{Ergebnisse und Diskussion}

Zur Kalibrierung wurden Proben, die $10-1000 \mathrm{mg} / \mathrm{l}$ Tri- $n$-butylphosphat in Chloroform und $10 \mu$ internen Standard enthielten, in den Gaschromatographen auf eine Durabond-Wax Kapillare gespritzt. Tri- $n$-butylphosphat eluierte nach 5,3 Minuten und Tri- $n$-pentylphosphat (interner Standard) nach 10,4 Minuten. Es zeigte sich eine lineare Beziehung zwischen den Peakflächen und den entsprechenden Substanzmengen.

Die Extraktion von Tri- $n$-butylphosphat und Tri- $n$ pentylphosphat von der $\mathrm{C}_{18}$-Säule wurde unter $\mathrm{An}$ wendung verschiedener Volumina unterschiedlicher Lösungsmittel optimiert. Dabei erwies sich die Elution mit $2 \times 300 \mu$ l Chloroform als am effektivsten. Zuvor mußte das verbliebene Wasser vollständig im Luftstrom aus der Säule entfernt werden. Der Vergleich direkt eingespritzter Standards mit denen, die dem Extraktionsprozeß unterzogen waren, ergab für Tri$n$-butylphosphat und Tri- $n$-pentylphosphat eine Ausbeute von über $90 \%$. Die Wiederfindungsrate blieb bei der Analyse präparierter Plasmaproben gleich.

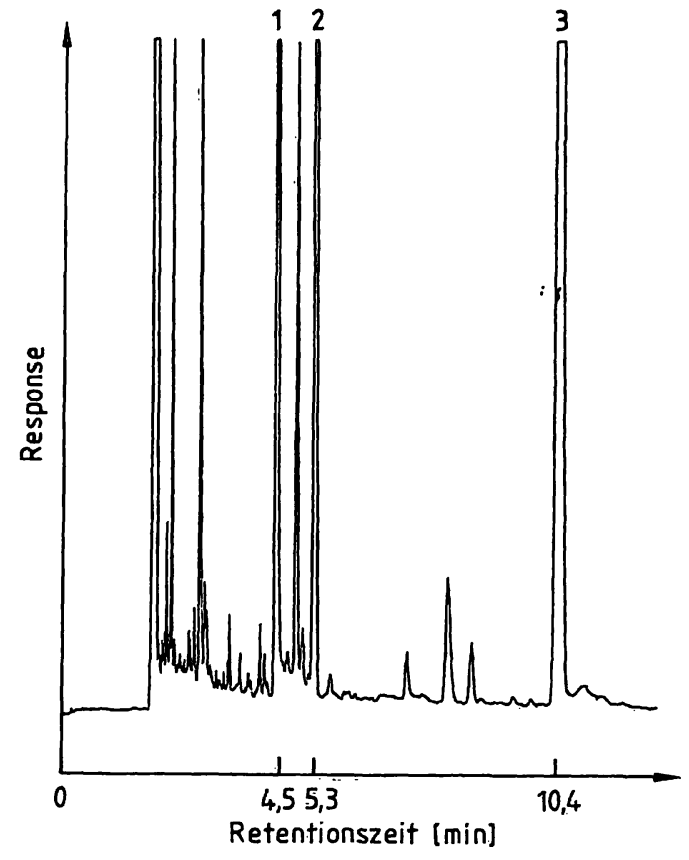

Abb. 1. Chromatogramm eines Serumpräparates, das Tri- $n$-butylphosphat (2), Caprylsäure (1) und Tri- $n$-pentylphosphat (3) enthielt.

Abbildung 1 zeigt das Chromatogramm einer aufgearbeiteten Serumprobe, die $10 \mathrm{mg} / \mathrm{l}$ Tri- $n$-butylphosphat enthielt. Neben Tri- $n$-butylphosphat und dem internen Standard Tri- $n$-pentylphosphat konnte noch Caprylsäure, die zur Stabilisierung von Albumin dient, nach 4,5 Minuten detektiert werden. Kontrollversuche zeigten keine signifikanten Interferenzen der genannten Verbindungen mit anderen Substanzen in den zu untersuchenden Proben.

Die Nachweisgrenze für Tri-n-butylphosphat liegt bei dieser Kombination aus Festphasenextraktion und Kapillar-Gaschromatographie bei etwa $50 \mu \mathrm{g} / \mathrm{l}$ Serum. Bei Einsatz einer wesentlich kostengünstigeren Weichglaskapillare (WG-11W) waren die Nachweisgrenze geringfügig schlechter, die Ergebnisse sonst aber nahezu gleichwertig.

Eine Flüssig-Flüssigextraktion verschiedener Proben nach Proteinfällung mit Perchlorsäure ähnlich der Methode von Horowitz et al. (6) ergab sowohl für den internen Standard Tri- $n$-pentylphosphat als auch für Tri- $n$-butylphosphat schlechtere Ausbeuten als mit dem hier beschriebenen Verfahren. Sie betrugen für den internen Standard etwa $80 \%$ und für Tri- $\dot{n}$-butylphosphat entsprechend korrigiert etwa $70 \%$ der eingesetzten Mengen (Tab. 1).

Somit erweist sich die Festphasenextraktion bei der Anreicherung von Tri- $n$-butylphosphat aus Blutpräparaten als eine einfache Methode mit hoher Wiederfindungsrate. Bei der Verwendung einer Kapillare für die gaschromatographische Untersuchung können unter isothermen Bedingungen Nachweisgrenzen erreicht werden, welche die üblichen Anforderungen weit übertreffen. 
Tab. 1. Wiederfindung von Tri-n-butylphosphat und Tri-n-pentylphosphat (inlerner Standard) aus 4 ml Serum nach verschiedenen Extraktionstechniken. Der relative Gehalt gibt don absoluten Gehalt, korrigiert um den Faktor des internen Standards, un.

\begin{tabular}{|c|c|c|c|c|c|}
\hline \multicolumn{4}{|c|}{ Tri-n-butylphosphat } & \multirow{2}{*}{\multicolumn{2}{|c|}{$\frac{\text { Tri-n-pentylphosphal }}{\text { absolut }}$}} \\
\hline \multicolumn{2}{|c|}{ absolut } & \multicolumn{2}{|c|}{ relativ } & & \\
\hline$\mu \mathrm{g}$ & $\%$ & $\mu g$ & $\%$ & $\mu \mathrm{g}$ & $\%$ \\
\hline $\begin{array}{l}40 \\
22,7 \\
37,6\end{array}$ & $\begin{array}{r}100 \\
57 \\
94\end{array}$ & $\begin{array}{l}40 \\
28 \\
38,4\end{array}$ & $\begin{array}{r}100 \\
70 \\
96\end{array}$ & $\begin{array}{r}100 \\
81 \\
98\end{array}$ & $\begin{array}{r}100 \\
81 \\
98\end{array}$ \\
\hline
\end{tabular}

\section{Literatur}

1. Shikata, T., Karasawa, T., Abc, K., Takahashi, T., Mayumi, M. \& Oda, T. (1978) J. Infect. Dis. 138, 242-244.

2. Hcimburger, V. N., Schwinn, H., Grotz, P., Luben, G., Kumpe. G. \& Herchenhan, B. (1981) Drug Res. 31, 619622.

3. Prince, A. M., Stephen, W. \& Brotman, B. (1983) Rev. Infect. Dis. 5, $92-107$.
4. Delcrmann, H. \& Joachim, H. U. (1971) \%. Klin. Cheın. Klin. Biochem. 9, 398-401.

5. Prince, A. M., Horowit\%, B., Huima, T., Richardson, L. \& van den Ende, M. C. (1984) Vox Sang. 46, 36-43.

6. Horowitz, B., Wiebe, M. E., Lippin, A. \& Stryker, M. IH. (1985) Transfusion 25, 516-522.

7. Prince, A. M., Horowit\%, B. \& Brotman, B. (1986) Lancet I, 706-710.

Dr. P. Preiffer

Institut für Mikrobiologic

und Weinforschung

Johannes Gutenberg-Universität Main\%.

Postfach 3980

D-6500 Mainz 
